\title{
Color Stability of Resin Used for Caries Infiltration After Exposure to Different Staining Solutions
}

\author{
AB Borges $\bullet$ TMF Caneppele $\bullet \mathrm{M} \mathrm{Luz}$ \\ CR Pucci • CRG Torres
}

\begin{abstract}
Clinical Relevance
Patients should be aware of minimizing the intake of colored staining solutions when submitted to resin infiltration treatment of white spot lesions in esthetically compromised areas.
\end{abstract}

\section{SUMMARY}

Purpose: The aim of this study was to investigate the staining behavior of demineralized enamel infiltrated by low-viscosity resin.

Methods and Materials: Bovine enamel/dentin cylindrical samples $(3 \times 2 \mathrm{~mm})$ were assigned into four groups $(n=45)$ according to the enamel treatment: sound enamel (control), deminer-

Alessandra Bühler Borges, DDS, PhD, São José dos Campos School of Dentistry, UNESP Univ. Estadual Paulista, Restorative Dentistry, São Paulo, Brazil

*Taciana M F Caneppele, DDS, MS, PhD, UNESP-Univ Estadual Paulista, São José dos Campos School of Dentistry, Restorative Dentistry, São Paulo, Brazil

Mariana Luz, UNESP-Univ Estadual Paulista, São José dos Campos School of Dentistry, Restorative Dentistry, São Paulo, Brazil

Cesar Rogério Pucci, São Joseì dos Campos Dental School, UNESP, Restorative Dentistry, São Paulo, Brazil

Carlos Rocha Gomes Torres, UNESP-Univ Estadual Paulista, São José dos Campos School of Dentistry, Restorative Dentistry, São Paulo, Brazil

*Corresponding author: Av. Francisco José Longo, 777, São José dos Campos, São Paulo 12245000, Brazil; e-mail: taciana@fosjc.unesp.br

DOI: $10.2341 / 13-150-\mathrm{L}$ alization + artificial saliva, demineralization + daily application of $0.05 \% \mathrm{NaF}$, demineralization + resin infiltration (Icon, DMG). Artificial white spot lesions were produced in groups with demineralization. After the treatments, color was assessed by spectrophotometry, using the CIE $L^{*} a^{*} b^{*}$ system. The specimens $(n=15)$ were then immersed in deionized water, red wine, or coffee for 10 minutes daily for eight days. Color was measured again, and the specimens were repolished with sandpaper discs. The final color was assessed. Data were analyzed by two-way analysis of variance and Tukey tests $(\alpha=0.05)$. A paired $t$-test was used for comparison between staining and repolishing conditions.

Results: There were significant differences for surface treatment and dye after staining and repolishing. Immersion in wine and coffee resulted in significantly increased color alteration $(\Delta E)$ compared with water $(p=0.001)$. The resin-infiltrated group exhibited the highest staining values $(p=0.001)$. The repolishing procedures resulted in significantly decreased color change. 
Conclusion: The exposure of specimens to colored solutions resulted in significant color alteration. The demineralized enamel treated with resin infiltration showed significantly higher staining than all other tested groups; however, the repolishing of the specimens minimized the staining effect.

\section{INTRODUCTION}

The caries lesion is a product of the dynamic process comprising several disorders of mineral balance between the tooth and the fluid of adjacent dental plaque, resulting in mineral loss. This loss can be reflected clinically in different ways from its first manifestation as enamel opacity (white spot), to large cavities that can extend up to the dental pulp. ${ }^{1}$

The active white spot is a consequence of an optical effect related to light scattering, due to differences in the refractive index of the involved components. A greater scattering is obtained when the porosities of demineralized enamel are filled with air, since its refractive index is 1.00 , lower than the refractive index of hydroxyapatite (1.62-1.65). This results in a whitish and opaque appearance of the enamel. If the enamel porosities of an early-stage lesion are filled with an aqueous solution showing the same refraction index of hydroxyapatite, the existing spaces are full and the enamel keeps its translucency. ${ }^{2}$

Since the stage of white spot does not involve enamel cavitation, noninvasive treatment with topical fluorides associated with diet and hygiene procedure orientation are recommended. However, the whitish appearance may remain, even if the lesion is arrested. This is because the remineralization of deeper lesions occurs only superficially, so that the body of the lesion remains porous and therefore still whitish, as a permanent scar. ${ }^{3,4}$

The presence of white spots in the anterior teeth can compromise esthetics, and it is frequent in orthodontic patients that do not perform oral hygiene adequately, as the excess of the material that promotes the adhesion between the orthodontic bracket and the tooth represents a critical factor for plaque accumulation. In addition, the cleaning action promoted by saliva, and chewing is reduced around the brackets. ${ }^{3}$ The cosmetic treatment for white spot lesions that remain as scars generally involves removal of affected tissue, by microabrasion of enamel ${ }^{5,6}$ or conventional restoration. ${ }^{7}$
The resin infiltration technique was recently introduced in dentistry to prevent further progression of enamel lesions. The objective is to fill the pores within the lesion body by capillary action, with a low-viscosity light-cured resin. This prevents further diffusion of bacteria, and consequently, lesion development, and establishes a barrier within the caries lesion, which can reinforce the enamel structure, avoiding or delaying cavitation and disruption of the surface. ${ }^{8,9}$ This technique requires no preparation or anesthesia and does not alter the anatomical shape of the tooth. ${ }^{10}$

Although this treatment was first introduced to arrest caries lesions, an additional positive effect was the esthetic improvement of anterior teeth when white spot lesions were present. This occurs due to the infiltration of the enamel porosities with the lowviscosity resin, which alters the refractive index of the light and consequently the final tooth appearance. Since the refractive index of the infiltrant resin (1.51) is close to hydroxyapatite, its ability to mask white spot lesions was observed. ${ }^{11-13}$

The resin-based materials are widely used in esthetic restorations, but they are subject to color alteration over time. The most common causes of discoloration are adsorption of dyes as a result of exposure to exogenous sources. ${ }^{14}$ The extent of discoloration varies according to the patient's habits, such as oral hygiene and diet. ${ }^{14-18}$

Whereas the resin infiltration technique seems promising for dentistry (and as previously mentioned, resins may suffer discoloration), this study investigated the staining behavior of low-viscosity resin used for infiltration. It also investigated the effect of repolishing in the color alteration of the stained specimens.

The null hypotheses tested were that the color of the demineralized enamel surface treated with topical fluoride and resin infiltration is not changed after exposure to dyes and that the polishing of the specimens after staining does not interfere with their color.

\section{METHODS AND MATERIALS}

\section{Specimen Preparation}

The methods described by Wiegand and others ${ }^{19}$ were used to prepare the specimens. For that, 90 extracted, nondamaged bovine incisors were stored in $0.1 \%$ thymol solution at room temperature until required. Enamel-dentin specimens, $3 \mathrm{~mm}$ in diameter and $2.2 \mathrm{~mm}$ in height, were prepared from the labial surface with a trephine mill. 
The specimens were positioned in a silicon mold with a cavity $6 \mathrm{~mm}$ in diameter and $2 \mathrm{~mm}$ in depth. On the bottom of the mold, there was a second-level cavity $3 \mathrm{~mm}$ in diameter and $0.1 \mathrm{~mm}$ depth, as described in a previous study. ${ }^{20}$ The specimens were placed inside the internal cavity with the enamel surface to the bottom of the mold. The mold was filled with low-viscosity composite resin (Oppalis Flow, FGM, Joinville, Brazil) and light cured for 40 seconds. On the side of the mold, there was a projection in the shape of a line that produced a lateral groove on the specimen that helped to achieve the correct position at the time of the color reading. The specimens were attached to a metal holder, and $0.1 \mathrm{~mm}$ of enamel was removed by polishing with sequential aluminum oxide abrasive papers (1200, 2400, and 4000 grit, FEPA-P, Struers, Ballerup, Denmark) in a polishing device (DP- 10, Panambra Industrial e Técnica SA, São Paulo, SP, Brazil) for 20 seconds each. The dentin side of specimens was abraded with a 1200 -grit abrasive paper, removing $0.1 \mathrm{~mm}$ of dentin and resulting in specimens of $1 \mathrm{~mm}$ of enamel and $1 \mathrm{~mm}$ of dentin. The prepared specimens were examined under the stereomicroscope to certify the absence of cracks or other surface defects. After preparation, the specimens were stored in $0.1 \%$ thymol solution to avoid dehydration.

Prior to treatment, the baseline $\mathrm{L}^{*}$ value of each specimen was assessed (M1) under standardized ambient conditions according to the Commission Internationale de l'Eclariage (CIE) $\mathrm{L}^{*} \mathrm{a}^{*} \mathrm{~b}^{*}$ system, using a spectrophotometer (CM2600d, Konica Minolta, Osaka, Japan). The device was adjusted to use the D65 standard light source with $100 \%$ UV and specular component included. The observer angle was set at $2^{\circ}$, and the device was adjusted to a small reading area (SAV). The color of each sample was measured three times and averaged. The results of color measurements were quantified in terms of three coordinate values $\left(L^{*}, a^{*}, b^{*}\right)$, as established by CIE, which locates the color of an object in a threedimensional color space. The $\mathrm{L}^{*}$ axis represents the degree of lightness within a sample and ranges from 0 (black) to 100 (white). The $\mathrm{a}^{*}$ plane represents the degree of green/red color, while the $b^{*}$ plane represents the degree of blue/yellow color within the sample.

According to the $\mathrm{L}^{*}$ value, a stratified allocation was performed among 12 groups $(n=15)$. In three groups, the enamel was kept sound and stored in daily changed artificial saliva throughout the study period (groups $\mathrm{SE} / \mathrm{n}=45$ ). In the other groups, artificial enamel caries lesions were created, as described below (groups $\mathrm{DE} / \mathrm{n}=135$ ).

\section{Specimen Demineralization}

Following the proposal of Queiroz and others, ${ }^{21}$ artificial enamel subsurface lesions were produced by individually immersing and storing the specimens in a buffer solution. The demineralizing solution was composed of $50 \mathrm{mM}$ acetate buffer solution containing $1.28 \mathrm{mM} \mathrm{Ca}\left(\mathrm{NO}_{3}\right)_{2} * 4 \mathrm{H}_{2} \mathrm{O}, 0.74 \mathrm{mM} \mathrm{NaH}_{2} \mathrm{PO}_{4} *$ $2 \mathrm{H}_{2} \mathrm{O}$, and 0.03 ppm $\mathrm{F}$ at $\mathrm{pH} 5.0$ for 16 hours. The specimens were immersed separately in the solution at $37^{\circ} \mathrm{C}$. The total volume of solution used was calculated using $2 \mathrm{~mL} / \mathrm{mm}^{2}$ of the enamel area. This method produces subsurface lesions with a mean depth of $43 \mu \mathrm{m} .^{22}$

\section{Experimental Design}

The demineralized groups were divided according to treatment of white spot lesions $(n=45)$ : AS (artificial saliva), specimens were stored in $5 \mathrm{~mL}$ of artificial saliva for four weeks, changed every day, and DF (0.05\% fluoride solution), specimens were immersed daily for one minute in $1 \mathrm{~mL}$ of $0.05 \%$ $\mathrm{NaF}$ solution for four weeks. The fluoride solution was manipulated in our laboratory. After the daily fluoride immersion, the specimens were rinsed with deionized water and stored in artificial saliva; RI (resin infiltration), specimens were resin infiltrated (Icon, DMG, Hamburg, Germany) and stored in artificial saliva for four weeks. The infiltration procedure was performed according to the manufacturer's instructions. A 15\% hydrochloric acid gel (Icon-Etch) was applied on the demineralized enamel surface for two minutes and then water rinsed and air dried for 30 seconds, followed by the application of ethanol (Icon-Dry) during 30 seconds and additional air drying. The low-viscosity resin infiltrant (Icon-Infiltrant) was applied on the surface two times, the first time for three minutes and the second time for one minute. Both applications were light cured for 40 seconds. Specimens were polished with aluminium oxide abrasive papers (4000 grit, FEPA-P; Struers) for 20 seconds for removal of the excess resin. A pilot study was conducted to verify the thickness of the specimens before and after the resin infiltration using a micrometer, and it was determined that the application of the 4000-grit paper for 20 seconds was adequate to remove the excess layer of infiltration resin.

Artificial saliva was prepared according to the formulation of Gohring and others. ${ }^{23}$ The $\mathrm{pH}$ was 


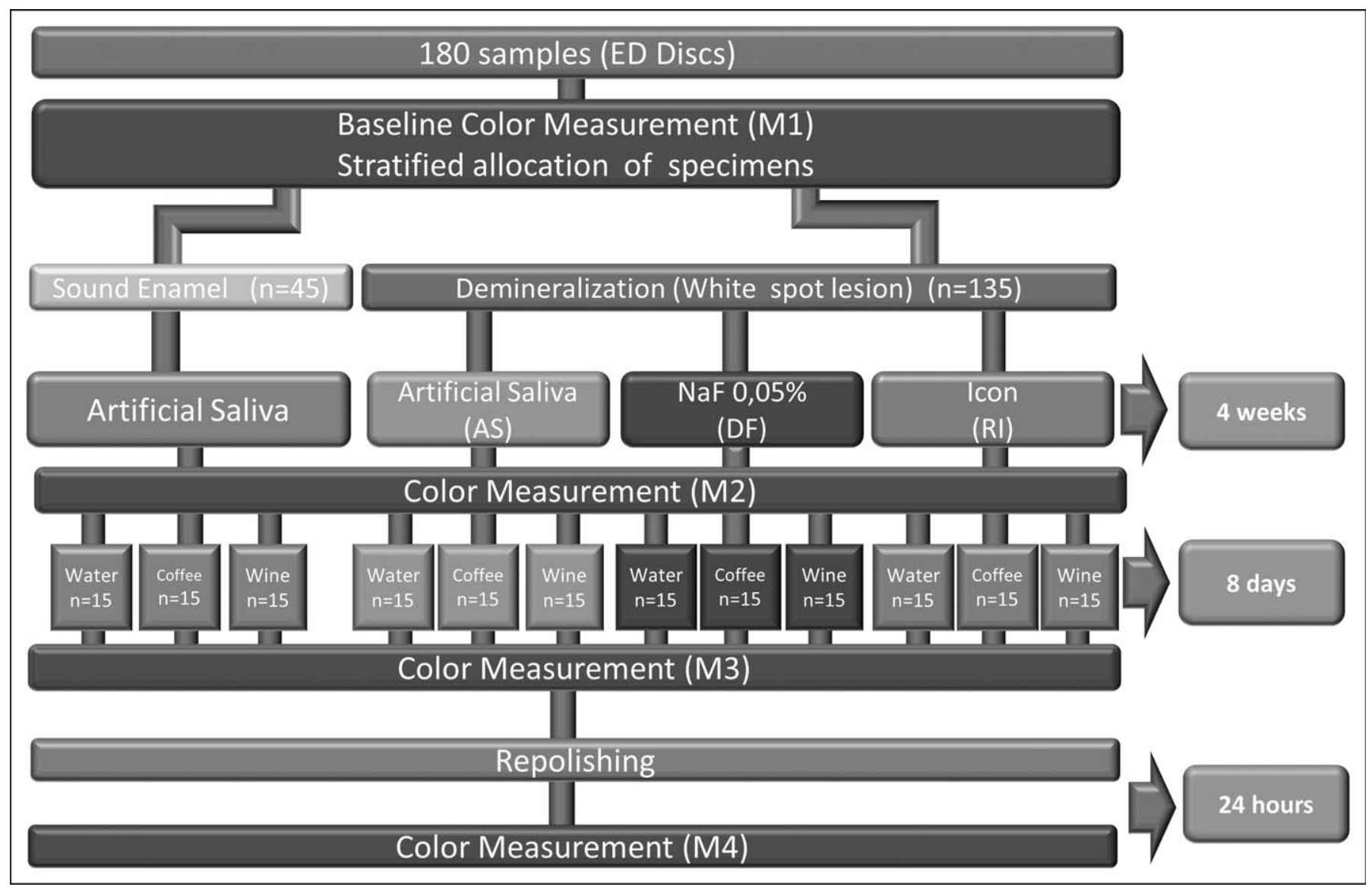

Figure 1. Experimental Design

adjusted to 7.0. After four weeks, the color was measured again (M2) for all samples, and then each group was divided into subgroups $(n=15)$ according to exposure to $250 \mathrm{~mL}$ of the following solutions: deionized water, soluble coffee solution prepared using $25 \mathrm{~g}$ of powder to $250 \mathrm{~mL}$ of water (Tradition Nescafé, Nestlé, Araras, SP, Brazil, pH = 5.0), and red wine (Santa Carolina, Merlot, Chile, $2010,12.3^{\circ}$ G.L., $\mathrm{pH}=3.4$ ).

The specimens remained immersed in the solutions mentioned above for 10 minutes daily during eight days and stored in artificial saliva in the intermediate periods. ${ }^{24}$ The solutions were changed at each exposure. After this period, the specimens were rinsed with deionized water and color measurement was performed (M3).

The surface of the specimens was then polished using fine-grained abrasive discs (FGM) for five seconds and immersion in deionized water for rehydration for 24 hours. After this period, the color was measured again (M4).
The differences in the values of $\mathrm{L}^{*}(\Delta \mathrm{L}), \mathrm{a}^{*}(\Delta \mathrm{a})$, and $b^{*}(\Delta b)$ were determined and the overall change in color $(\Delta \mathrm{E})$ was calculated using the following formula:

$$
\begin{aligned}
\Delta E_{1}= & {\left[\left(L_{M 3} *-L_{M 2} *\right)^{2}+\left(a_{M 3} *-a_{M 2} *\right)^{2}\right.} \\
& \left.+\left(b_{M 3} *-b_{M 2} *\right)^{2}\right]^{0,5} \\
\Delta E_{2}= & {\left[\left(L_{M 4} *-L_{M 2} *\right)^{2}+\left(a_{M 4} *-a_{M 2} *\right)^{2}\right.} \\
& \left.+\left(b_{M 4} *-b_{M 2} *\right)^{2}\right]^{0,5}
\end{aligned}
$$

Figure 1 shows the experimental design.

\section{Statistical Analysis}

Data were statistically analyzed using two-way analysis of variance and Tukey's tests for color measurement after staining and after repolishing. A paired $t$-test was conducted to evaluate if the repolishing procedures influenced the specimens 


\begin{tabular}{|c|c|c|c|c|c|c|c|c|}
\hline \multirow[t]{2}{*}{ Group } & \multicolumn{2}{|c|}{ Sound Enamel } & \multicolumn{2}{|c|}{ White Spot Lesion + AS } & \multicolumn{2}{|c|}{ White Spot Lesion + DF } & \multicolumn{2}{|c|}{ White Spot Lesion + RI } \\
\hline & $\Delta \mathrm{E} 1^{\mathrm{a}}$ & $\Delta \mathrm{E} 2^{\mathrm{b}}$ & $\Delta \mathrm{E} 1^{\mathrm{a}}$ & $\Delta \mathrm{E} 2^{\mathrm{b}}$ & $\Delta \mathrm{E} 1^{\mathrm{a}}$ & $\Delta \mathrm{E} 2^{\mathrm{b}}$ & $\Delta \mathrm{E} 1^{\mathrm{a}}$ & $\Delta \mathrm{E} 2^{\mathrm{b}}$ \\
\hline Wine & $14.4^{*}(4.2) \mathrm{cd}$ & $10.1^{*}(2.2) \mathrm{C}$ & $11.1^{*}(5.1) \mathrm{bc}$ & $9.3^{*}(5.4) \mathrm{C}$ & $14.2^{*}(2.7) \mathrm{cd}$ & $8.4^{*}(3.2) \mathrm{C}$ & $17.3^{*}(2.7) \mathrm{de}$ & $14.7^{*}(4.7) \mathrm{D}$ \\
\hline Coffee & $14.5^{\star}(4.4) \mathrm{cd}$ & $8.1^{*}(4.5) \mathrm{C}$ & $9.5^{\star}(5.9) b$ & $8.4^{*}(5.7) \mathrm{C}$ & $7.3^{*}(2.3) b$ & $6.0^{*}(1.4) \mathrm{BC}$ & $21.3^{*}(4.3) \mathrm{e}$ & $16.6^{*}(4.3) \mathrm{D}$ \\
\hline
\end{tabular}

color, for each experimental condition. The significance level used was $p<0.05$.

\section{RESULTS}

The overall color change of the specimens after dye immersion $\left(\Delta \mathrm{E}_{1}\right)$ and after repolishing procedures $\left(\Delta \mathrm{E}_{2}\right)$ for all experimental conditions is shown in Table 1. The cross-product surface treatment vs dye as well as the factors surface treatment and dye were statistically significant $(p=0.001)$.

The paired $t$-test compared color changes after dye immersion $(\Delta \mathrm{E} 1)$ and after repolishing $(\Delta \mathrm{E} 2)$, group by group. For all experimental conditions with coffee or wine immersion, the repolishing procedures resulted in significantly decreased color change (Table 1).

Table 2 shows the results of the surface treatment as a factor. The color change after dye immersion and after repolishing was significantly higher for the white spot lesion + resin infiltration group.

The results of the Tukey test for the dye factor are shown in Table 3. The immersion in coffee and wine resulted in a significant color alteration compared with water exposure. Analysis of $\mathrm{L}^{*} \mathrm{a}^{*} \mathrm{~b}^{*}$ values showed decreasing lightness values $(\Delta \mathrm{L})$ for coffee and wine, whereas $\Delta$ a remained stable. The specimens immersed in the coffee solution exhibited an increase in $\Delta \mathrm{b}$.

\section{DISCUSSION}

Several methods can be used to evaluate tooth color with visual comparison with tooth color shade guides, image analysis, colorimeters, or spectrophotometers. ${ }^{18,25}$ Since spectrophotometers allow an objective color assessment and provide precise quantitative data, this was the method used in the present study. ${ }^{26,27}$ The spectrophotometer presents $\mathrm{L}^{*}, \mathrm{a}^{*}$, and $\mathrm{b}^{*}$ values; therefore, as the staining solutions alter the specimens color in all axis, the parameter $\Delta \mathrm{E}$ was used as a response variable, because it indicates the color change of specimens at two different moments, allowing one to evaluate the overall color change. ${ }^{12,27}$ In the present study, the most affected parameter was $\mathrm{L}^{*}$, indicating a reduced lightness of stained specimens.

Because of the difficulties in obtaining human teeth with the ideal characteristics for the experiment, bovine incisors were used, providing discs with standardized enamel/dentin thickness. The chemical and physical properties of bovine substrate, such as composition, density, and microhardness, are very similar to human enamel. ${ }^{28}$ Bovine and human substrates are also found to have a similar behavior regarding staining effects. ${ }^{29}$

Tooth color perception is associated with scattering of incident light on the tooth structure and its absorption along this way. ${ }^{30}$ The initial enamel caries lesions is characterized by a whitish appearance, due to changes of the refractive index of the light in the demineralized tissue compared with the surrounding sound enamel, ${ }^{31}$ causing an undesirable esthetic appearance when present in the anterior teeth.

When properly treated with preventive measures, the white spot lesions may become arrested and exhibit a smooth and shiny appearance. In the remineralization process, saliva plays an important role, providing minerals that will partially replace the mineral lost during the demineralization process. ${ }^{31}$ Nevertheless, during this remineralization phase, dyes can be incorporated into the lesions,

\begin{tabular}{|lcc|}
\hline Table 2: & $\begin{array}{l}\text { Mean and Standard Deviation (SD) Data and } \\
\text { Results of Tukey Test for Surface Treatment for } \\
\Delta E_{1} \text { and } \Delta E_{2}\end{array}$ & \\
\hline Surface Treatment & $\begin{array}{c}\text { After Staining } \\
\left(\Delta \mathrm{E}_{1}\right)^{*}\end{array}$ & $\begin{array}{c}\text { After Repolishing } \\
\left(\Delta \mathrm{E}_{2}\right)^{*}\end{array}$ \\
\hline Sound enamel (control) & $10.43(6.70) \mathrm{b}$ & $6.80(4.45) \mathrm{a}$ \\
\hline White spot lesion + AS & $7.85(5.77) \mathrm{a}$ & $6.57(5.58) \mathrm{a}$ \\
\hline White spot lesion + DF & $8.07(5.32) \mathrm{a}$ & $5.33(3.59) \mathrm{a}$ \\
\hline White spot lesion + RI & $13.63(8.83) \mathrm{c}$ & $11.13(7.43) \mathrm{b}$ \\
\hline${ }^{*}$ Different letters imply significant difference $(\mathrm{p}=0.001)$ among the groups. \\
\hline
\end{tabular}




\begin{tabular}{|c|c|c|c|c|c|c|c|c|}
\hline \multirow[t]{2}{*}{ Dye } & \multicolumn{4}{|c|}{ After Staining } & \multicolumn{4}{|c|}{ After Repolishing } \\
\hline & $\Delta \mathrm{L}$ & $\Delta \mathrm{a}$ & $\Delta \mathrm{b}$ & $\Delta \mathrm{E}_{1}{ }^{\mathrm{a}}$ & $\Delta \mathrm{L}$ & $\Delta \mathrm{a}$ & $\Delta \mathrm{b}$ & $\Delta \mathrm{E}_{2}^{\mathrm{b}}$ \\
\hline Coffee & $-9.94(5.67)$ & $1.16(1.17)$ & $8.34(4.25)$ & $13.16(6.92) b$ & $-8.06(4.90)$ & $0.49(0.86)$ & $5.18(3.59)$ & $9.78(5.83) \mathrm{B}$ \\
\hline Wine & $-13.22(4.62)$ & $1.36(0.81)$ & $-4.19(2.40)$ & $14.25(4.33) \mathrm{b}$ & $-10.27(4.77)$ & $0.66(0.67)$ & $-1.60(1.65)$ & 10.59 (4.69)B \\
\hline
\end{tabular}

resulting in the formation of unesthetic brown spots. In this study, the staining of the demineralized enamel groups after exposure to saliva and fluoride as remineralizing agents and immersion in dyes was observed. In general, immersion in coffee and wine promoted significant color change of specimens, regardless of the type of treatment of white spot lesion. Therefore, the first null hypothesis was rejected.

Coffee and red wine were chosen as dye testing substances because they are frequently consumed. ${ }^{32}$ Coffee exhibits a strong potential for staining both tooth structure and resinous materials. ${ }^{17,33}$ The compatibility between the brown dye of the coffee and the resin polymer chain has been suggested, facilitating the adsorption and penetration of the dye in the resin. ${ }^{33}$ The specimens immersed in coffee exhibited a yellow appearance, besides reduced lightness. On the other hand, the specimens immersed in wine presented a decrease in $b^{*}$ values. The complex combination of pigments present in the wine solution makes the prediction of changes on $\mathrm{a}^{*}$ and $b^{*}$ parameters difficult. Perhaps the purple pigments interfere with the $b^{*}$ coordinate, directing it toward blue. Moreover, the presence of alcohol and low $\mathrm{pH}$ could promote softening of the polymeric material, ${ }^{34}$ which could make the adsorption of pigments on the resin surface easier, such as tannins present in red wine. ${ }^{35,36}$

The susceptibility to extrinsic staining of a resinous material may also be related to the type of resin matrix used, due to its rate of water sorption and solubility. The absorption of water and other fluids by the organic component of resin can result in discoloration and degradation of the polymer matrix. $^{37}$

A high penetration coefficient and low viscosity of resin monomer are desirable features to allow infiltration of resin into the subsurface of carious lesions. The combination of experimental TEGDMA, HEMA, and ethanol showed satisfactory characteristics for an infiltrating resin. ${ }^{38}$ However, HEMA has a hydroxyl functional group, making it hydrophilic. ${ }^{37}$ Thus, although the composition of the infiltrating resin (ICON) is not detailed by the manufacturer, it may favor the absorption of waterbased dyes and increase the susceptibility of the material to staining. In addition, the inhibition of polymerization by oxygen and polymerization shrinkage of the infiltrating resin may also result in nonhomogeneous areas, ${ }^{37}$ which could facilitate the penetration or adsorption of the dyes. Nevertheless, a recent study reported that the polishing of infiltrated lesions increases their resistance to staining challenges. ${ }^{12}$

The second null hypothesis was also rejected, since repolishing with fine-grain abrasive discs led to a significant reduction in $\Delta \mathrm{E}$ values for all groups. Similar results were also observed in a previous study with composite resins, ${ }^{32}$ and this is due to the fact that the dyes can remain adsorbed on the surface, with little penetration into the resinous materials or the tooth substrate. ${ }^{17}$

However, even after repolishing, the stained specimens still presented color alteration significantly higher than those immersed in deionized water. Overall, those subjected to the resin infiltration technique exhibited $\Delta \mathrm{E}$ values significantly higher than all other groups. Nevertheless, it should be pointed out that the combination of immersion time in the dyes and absence of tooth brushing probably increased the staining susceptibility. ${ }^{37}$ In clinical conditions, the dilution of the dye solutions by saliva and the action of brushing associated with the use of toothpastes can result in a greater resistance of the infiltrating resin to staining. Nonetheless, patients should be warned about the potential for staining by food dyes and avoid or minimize the consumption of such colored foods to increase the longevity of the esthetic outcomes promoted by the treatment of white spot lesions with infiltration resin in anterior teeth.

\section{CONCLUSIONS}

Based on the methodology used and considering the limitations of this study, it can be concluded that the 
immersion of the specimens in staining solutions (wine and coffee) resulted in significant color alteration. The demineralized enamel treated with resin infiltration showed significantly higher staining than all other tested groups; however, repolishing of the specimens can minimize the staining effect.

\section{Acknowledgement}

This study was supported by the State of São Paulo Research Foundation-FAPESP (10/16850-5).

\section{Conflict of Interest}

The authors of this article certify that they have no proprietary, financial, or other personal interest of any nature or kind in any product, service, and/or company that is presented in this article.

(Accepted 13 August 2013)

\section{REFERENCES}

1. Fejerskov O, \& Kidd E (2009) Dental Caries: The Disease and Its Clinical Management. 2nd ed. Blackwell Munksgaard, Oxford, UK.

2. Houwink B (1974) The index of refraction of dental enamel apatite British Dental Journal 137(12) 472-475.

3. Mattousch TJ, van der Veen MH, \& Zentner A (2007) Caries lesions after orthodontic treatment followed by quantitative light-induced fluorescence: a 2-year followup European Journal of Orthodontics 29(3) 294-298.

4. Zantner C, Martus P, \& Kielbassa AM (2006) Clinical monitoring of the effect of fluorides on long-existing white spot lesions Acta Odontologica Scandinavica 64(2) 115-122.

5. Ardu S, Castioni NV, Benbachir N, \& Krejci I (2007) Minimally invasive treatment of white spot enamel lesions Quintessence International 38(8) 633-636.

6. Croll TP (1989) Enamel microabrasion for removal of superficial discoloration Journal of Esthetic Dentistry 1(1) $14-20$.

7. Malterud MI (2006) Minimally invasive restorative dentistry: a biomimetic approach Practical Procedures \& Aesthetic Dentistry 18(7) 409-414.

8. Phark JH, Duarte S Jr, Meyer-Lueckel H, \& Paris S (2009) Caries infiltration with resins: a novel treatment option for interproximal caries Compendium of Continuing Education in Dentistry 30(Spec No) 313-317.

9. Paris S, \& Meyer-Lueckel H (2010) Inhibition of caries progression by resin infiltration in situ Caries Research 44(1) 47-54.

10. Kugel G, Arsenault P, \& Papas A (2009) Treatment modalities for caries management, including a new resin infiltration system Compendium of Continuing Education in Dentistry 30(Spec No) 31-10.

11. Paris S, \& Meyer-Lueckel H (2009) Masking of labial enamel white spot lesions by resin infiltration-a clinical report Quintessence International 40(9) 713-718.
12. Paris S, Schwendicke F, Keltsch J, Dorfer C, \& MeyerLueckel H. Masking of white spot lesions by resin infiltration in vitro. Journal of Dentistry Prepublished Apr 11, 2013. doi:pii: S0300-5712(13)00094-8

13. Rocha Gomes Torres C, Borges AB, Torres LM, Gomes IS, \& de Oliveira RS (2011) Effect of caries infiltration technique and fluoride therapy on the colour masking of white spot lesions Journal of Dentistry 39(3) 202-207.

14. Um CM, \& Ruyter IE (1991) Staining of resin-based veneering materials with coffee and tea Quintessence International 22(5) 377-386.

15. Tunc ES, Bayrak S, Guler AU, \& Tuloglu N (2009) The effects of children's drinks on the color stability of various restorative materials Journal of Clinical Pediatric Dentistry 34(2) 147-150.

16. Topcu FT, Sahinkesen G, Yamanel K, Erdemir U, Oktay EA, \& Ersahan S (2009) Influence of different drinks on the colour stability of dental resin composites European Journal of Dentistry 3(1) 50-56.

17. Chan KC, Hormati AA, \& Kerber PE (1981) Staining calcified dental tissues with food Journal of Prosthetic Dentistry 46(2) 175-178.

18. Joiner A (2004) Tooth colour: a review of the literature Journal of Dentistry 32(Supplement 1) 3-12.

19. Wiegand A, Vollmer D, Foitzik M, Attin R, \& Attin T (2005) Efficacy of different whitening modalities on bovine enamel and dentin Clinical Oral Investigations 9(2) 91-97.

20. Torres CR, Wiegand A, Sener B, \& Attin T (2010) Influence of chemical activation of a $35 \%$ hydrogen peroxide bleaching gel on its penetration and efficacyin vitro study Journal of Dentistry 38(10) 838-846.

21. Queiroz CS, Paes Hara AT, Leme AF, \& Cury JA (2008) $\mathrm{pH}$-cycling models to evaluate the effect of low fluoride dentifrice on enamel de- and remineralization Brazilian Dental Journal 19(1) 21-27.

22. Magalhaes AC, Moron BM, Comar LP, Wiegand A, Buchalla W, \& Buzalaf MA (2009) Comparison of crosssectional hardness and transverse microradiography of artificial carious enamel lesions induced by different demineralising solutions and gels Caries Research 43(6) 474-483.

23. Gohring TN, Zehnder M, Sener B, \& Schmidlin PR (2004) In vitro microleakage of adhesive-sealed dentin with lactic acid and saliva exposure: a radio-isotope analysis Journal of Dentistry 32(3) 235-240.

24. Attin T, Manolakis A, Buchalla W, \& Hannig C (2003) Influence of tea on intrinsic colour of previously bleached enamel Journal of Oral Rehabilitation 30(5) 488-494.

25. Chu SJ, Trushkowsky RD, \& Paravina RD (2010) Dental color matching instruments and systems: review of clinical and research aspects Journal of Dentistry 38(Supplement 2) e2-e16.

26. Horn DJ, Bulan-Brady J, \& Hicks ML (1998) Sphere spectrophotometer versus human evaluation of tooth shade Journal of Endodontics 24(12) 786-790.

27. Corekci B, Irgin C, Malkoc S, \& Ozturk B (2010) Effects of staining solutions on the discoloration of orthodontic 
adhesives: an in-vitro study American Journal of Orthodontics and Dentofacial Orthopedics 138(6) 741-746.

28. Esser M, Tinschert J, \& Marx R (1998) Material characteristics of the hard tissues of bovine versus human teeth [in German] Deutsche zahnärztliche Zeitschrift 53(10) 713-717.

29. Attia ML, Aguiar FH, Mathias P, Ambrosano GM, Fontes CM, \& Liporoni PC (2009) The effect of coffee solution on tooth color during home bleaching applications American Journal of Dentistry 22(3) 175-179.

30. ten Bosch JJ, \& Coops JC (1995) Tooth color and reflectance as related to light scattering and enamel hardness Journal of Dental Research 74(1) 374-380.

31. Featherstone JD (1999) Prevention and reversal of dental caries: role of low level fluoride Community Dentistry and Oral Epidemiology 27(1) 31-40.

32. Anfe TE, Agra CM, \& Vieira GF (2011) Evaluation of the possibility of removing staining by repolishing composite resins submitted to artificial aging Journal of Esthetic and Restorative Dentistry 23(4) 260-267.

33. Park JK, Kim TH, Ko CC, Garcia-Godoy F, Kim HI, \& Kwon YH (2010) Effect of staining solutions on discolor- ation of resin nanocomposites American Journal of Dentistry 23(1) 39-42.

34. Asmussen E, \& Peutzfeldt A (2001) Influence of pulsedelay curing on softening of polymer structures Journal of Dental Research 80(6) 1570-1573.

35. Ardu S, Braut V, Gutemberg D, Krejci I, Dietschi D, \& Feilzer AJ (2010) A long-term laboratory test on staining susceptibility of esthetic composite resin materials Quintessence International 41(8) 695-702.

36. Asmussen E, \& Hansen EK (1986) Surface discoloration of restorative resins in relation to surface softening and oral hygiene Scandinavian Journal of Dental Research 94(2) 174-177.

37. Bagheri R, Burrow MF, \& Tyas M (2005) Influence of food-simulating solutions and surface finish on susceptibility to staining of aesthetic restorative materials Journal of Dentistry 33(5) 389-398.

38. Paris S, Meyer-Lueckel H, Colfen H, \& Kielbassa AM (2007) Resin infiltration of artificial enamel caries lesions with experimental light curing resins Dental Materials Journal 26(4) 582-588. 